\title{
Scale size and life time of energy conversion regions observed by Cluster in the plasma sheet
}

\author{
M. Hamrin ${ }^{1}$, P. Norqvist ${ }^{1}$, O. Marghitu ${ }^{2}$, A. Vaivads ${ }^{3}$, B. Klecker ${ }^{4}$, L. M. Kistler ${ }^{5}$, and I. Dandouras ${ }^{6}$ \\ ${ }^{1}$ Department of Physics, Umeå University, Umeå, Sweden \\ ${ }^{2}$ Institute for Space Sciences, Bucharest, Romania \\ ${ }^{3}$ Swedish Institute of Space Physics, Uppsala, Sweden \\ ${ }^{4}$ Max-Planck-Institut für extraterrestrische Physik, Garching, Germany \\ ${ }^{5}$ Space Science Center, University of New Hampshire, Durham, USA \\ ${ }^{6}$ CESR-CNRS, Toulouse, France
}

Received: 11 June 2009 - Revised: 21 September 2009 - Accepted: 21 October 2009 - Published: 4 November 2009

\begin{abstract}
In this article, and in a companion paper by Hamrin et al. (2009) [Occurrence and location of concentrated load and generator regions observed by Cluster in the plasma sheet], we investigate localized energy conversion regions (ECRs) in Earth's plasma sheet. From more than 80 Cluster plasma sheet crossings ( $660 \mathrm{~h}$ data) at the altitude of about $15-20 R_{E}$ in the summer and fall of 2001, we have identified 116 Concentrated Load Regions (CLRs) and 35 Concentrated Generator Regions (CGRs). By examining variations in the power density, $\boldsymbol{E} \cdot \boldsymbol{J}$, where $\boldsymbol{E}$ is the electric field and $\boldsymbol{J}$ is the current density obtained by Cluster, we have estimated typical values of the scale size and life time of the CLRs and the CGRs. We find that a majority of the observed ECRs are rather stationary in space, but varying in time. Assuming that the ECRs are cylindrically shaped and equal in size, we conclude that the typical scale size of the ECRs is $2 R_{E} \lesssim \Delta S_{\mathrm{ECR}} \lesssim 5 R_{E}$. The ECRs hence occupy a significant portion of the mid altitude plasma sheet. Moreover, the CLRs appear to be somewhat larger than the CGRs. The life time of the ECRs are of the order of 1-10 min, consistent with the large scale magnetotail MHD simulations of Birn and Hesse (2005). The life time of the CGRs is somewhat shorter than for the CLRs. On time scales of 1-10 min, we believe that ECRs rise and vanish in significant regions of the plasma sheet, possibly oscillating between load and generator character. It is probable that at least some of the observed ECRs oscillate energy back and forth in the plasma sheet instead of channeling it to the ionosphere.
\end{abstract}

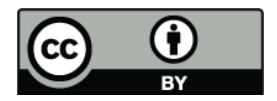

Correspondence to: M. Hamrin (hamrin@space.umu.se)
Keywords. Magnetospheric physics (Auroral phenomena; Magnetosphere-ionosphere interactions; Plasma sheet)

\section{Introduction}

It is well known that the plasma sheet, on the average, behaves as a load. Conversion from electromagnetical to mechanical energy occurs in load regions where the power density $\boldsymbol{E} \cdot \boldsymbol{J}>0$, where $\boldsymbol{E}$ is the electric field and $\boldsymbol{J}$ the current density. The process is reversed in generator regions where $\boldsymbol{E} \cdot \boldsymbol{J}<0$. Birn and Hesse (2005) investigated energy release, conversion and transport processes in the magnetotail and plasma sheet in a large scale MHD simulation. The simulation confirms the overall load behaviour of the plasma sheet, but also indicates that the plasma sheet is highly structured on a smaller scale with energy conversion occurring in both directions.

In a companion paper, Hamrin et al. (2009) [Occurrence and location of concentrated load and generator regions observed by Cluster in the plasma sheet], hereafter referred to as H09A, in situ data are used for investigating energy conversion in the plasma sheet. By evaluating the power density, $\boldsymbol{E} \cdot \boldsymbol{J}$, the local energy conversion is analyzed. Using $660 \mathrm{~h}$ of power density data derived from Cluster from the summer and fall of 2001, when the spacecraft probed the plasma sheet at an altitude of mainly $15-20 R_{E}$ (cf. Fig. 5 in H09A), 151 Energy Conversion Regions (ECRs) are identified and their location and occurrence are investigated. Hence, there are more load regions than generator regions. Out of the 151 ECRs, 116 ECRs are found to be Concentrated Load Regions (CLRs) while only 35 are Concentrated Generator Regions

Published by Copernicus Publications on behalf of the European Geosciences Union. 
(CGRs). Moreover, the loads also appear to be stronger. To our knowledge, these are the first in situ observations of the general load behaviour of the plasma sheet.

However, as indicated in the large scale MHD simulations by Birn and Hesse (2005), on a smaller scale the plasma sheet is highly structured, with energy conversion between the particles and the fields going in both directions. In the simulation, generator regions are found off the equatorial plane. Similar to this, in H09A we find that CGRs often prefer locations closer to the Plasma Sheet Boundary Layer (PSBL), while CLRs are located closer to the central plasma sheet.

The ECRs previously investigated (Marghitu et al., 2006, 2009; Hamrin et al., 2006), as well as the ECRs discussed here and in the companion paper H09A, are labelled concentrated load or generator regions (CLRs and CGRs) to distinguish them from more extended energy conversion regions which may also exist in the plasma sheet. However, investigating such extended structures is out of the scope of this article. The time it takes for Cluster to cross the plasma sheet (typically in the GSE z-direction) is of the order of several hours, while the ECRs are observed during much shorter times (of the order of minutes). An event study in Marghitu et al. (2006) suggests that a CGR might well be concentrated in both time and space, and a lower limit of the CGR extension along the field line was estimated to about $1000 \mathrm{~km}$.

Up till now, no experimental efforts have been made to try to characterize the scale size and life time of the CLRs and CGRs observed. It is not known whether the ECRs are spatial entities moving around and crossing the spacecraft path, or if they are a consequence of time variations in the power density with e.g. alternately oscillating load and generator characteristics. The Birn and Hesse (2005) simulations indicate that the processes of energy conversion in the plasma sheet boundary region indeed are not stationary in time. As shown in Fig. 9 of Birn and Hesse (2005), at a specific location in the plasma sheet, the power density varies with time. In their simulations they observed oscillations between generator and load character with periods of about 4 min which corresponds to about $2 \mathrm{~min}$ for loads and generators, respectively.

In this paper we use the data base from H09A to try to estimate time and size scales of the ECRs. An automatic event selection routine is used for identifying the ECR events from more than 80 Cluster plasma sheet crossings in the summer and fall of 2001. This data base allows for an extended survey of ECRs' properties in the mid altitude plasma sheet, and it offers the possibility of obtaining typical time and scale sizes of the CLRs and CGRs.

In this article we will show that the scale sizes of the ECRs are about one order of magnitude larger than the Cluster tetrahedron and that their life time is one or a few minutes which is consistent with Birn and Hesse (2005). We also argue that it is probable that the CLRs and CGRs observed in the plasma sheet data from 2001 are the consequence of the power density varying in time rather than in space.

\section{Instrumentation and method}

In this article and in the companion paper H09A, we use data from the Cluster ion spectrometer (CIS), the flux-gate magnetometer (FGM) and the electric fields and waves experiment (EFW) on board the Cluster spacecraft. For a discussion of the Cluster mission and instruments, see Escoubet et al. (2001) and references therein.

The evaluation of the power density, $\boldsymbol{E} \cdot \boldsymbol{J}$, is based on electric field and current density data re-sampled every $4 \mathrm{~s} \mathrm{(cf.}$ H09A). The electric field is derived from the two CIS instruments CODIF (Composition and Distribution Function) and HIA (Hot Ion Analyzer) on the assumption that the $\boldsymbol{E} \times \boldsymbol{B}$ drift is dominant. The electric field from the EFW instrument is used for cross-checking the results from CIS. The current density is obtained from simultaneous magnetic field measurements from the FGM instrument on board the four satellites by using the curlometer method, $\mathbf{J}=\nabla \times \mathbf{B} / \mu_{0}$ (Robert et al., 1998; Dunlop et al., 2002).

Since the current density, $\langle\boldsymbol{J}\rangle$, can be interpreted as an average over the Cluster tetrahedron, in most cases we use the electric field, $\langle\boldsymbol{E}\rangle$, averaged over all available spacecraft when computing the power density (CODIF is operational on Cluster spacecraft $\mathrm{C} 1, \mathrm{C} 3$, and $\mathrm{C} 4$, and HIA on $\mathrm{C} 1$ and C3). However, if not stated otherwise, in the following we simplify the notation by omitting the brackets around $\boldsymbol{E}$ and $J$.

To better analyze the scale size of the ECRs, we sometimes need to resolve each satellite's closeness to the edge of the ECRs. In some cases, one or a few satellites might in fact be localized outside the ECRs, hence reducing the ECR signature in the power density averaged over the spacecraft. To resolve the closeness of the satellites to the edge of the ECRs, we compute the power density by using the electric field measured by a single spacecraft only. This procedure can be motivated by the fact that the current density, obtained via the curlometer method, is representative for all satellites. The electric field, on the other hand, can be measured on each satellite and hence has a higher spatial resolution. In this article, and in the companion paper H09A, we clearly state whenever the electric fields from single spacecraft are used instead of an average over all available satellites.

To identify ECRs in the Cluster power density data, we use an automatic selection routine which identifies regions with clear and concentrated signatures of $\boldsymbol{E} \cdot \boldsymbol{J}>0$ and $\boldsymbol{E} \cdot \boldsymbol{J}<0$ for CLRs and CGRs, respectively. In Fig. 1 we show a schematic CLR. The region is highlighted in yellow and it manifests itself as a concentrated region with $\boldsymbol{E} \cdot \boldsymbol{J}>0$ above the surrounding fluctuations as shown in the top panel. The bottom panel contains the power density integrated over time along the spacecraft path. The CLR corresponds to the region with the clear step in the integrated power density. For a CGR the picture is similar, but $\boldsymbol{E} \cdot \boldsymbol{J}<0$ and the step is negative.

To be accepted by the automatic selection routine, every CLR and CGR must fulfill a set of instrumental and physical 

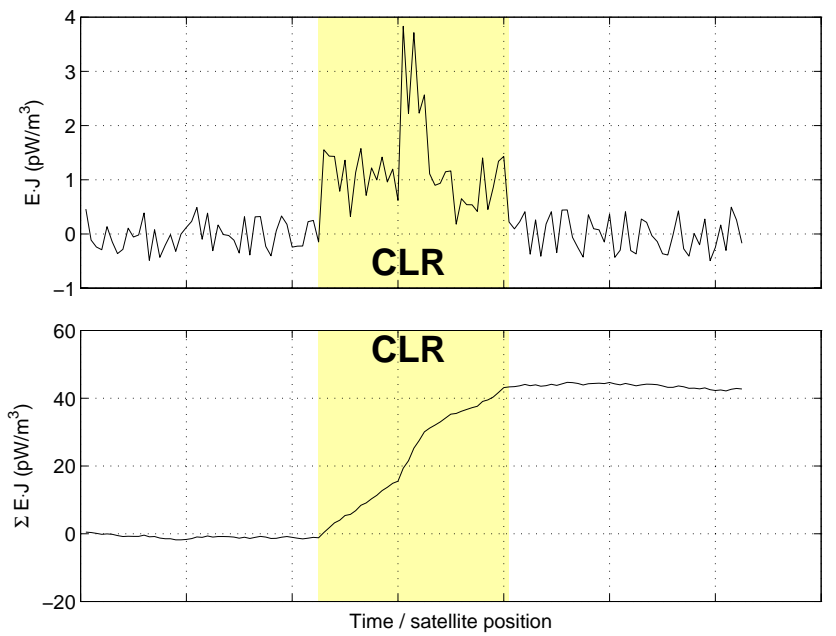

Fig. 1. Schematic CLR showing typical quantities discussed in this article. Top panel: power density measured by the satellites. Bottom panel: power density integrated over time along the spacecraft path. The CLR corresponds to the region of large $\mathbf{E} \cdot \mathbf{J}>0$ and the clear step in the integrated power density. The behaviour of a CGR is similar, but in this case the power density and step are negative.

criteria to assure a reliable selection. The routine is based on three separate steps. In the first step (1. Selection) we identify possible ECR events from the slope of the time integral of the power density along the satellite path. Only regions with large enough slopes are kept. However, many of the identified regions from the first step are very short, and they can be located close to each other, perhaps with some small noisy regions in between. Therefore we need a second step (2. Merging) where CLRs are merged with neighboring CLRs, and CGRs with neighbouring CGRs. In the final step (3. Rejection), all ECRs which do not satisfy a set of physical and instrumental requirements are rejected. For example, to ensure that the protons behave collectively within the ECRs, the selected regions must be large enough (a few proton gyroradii). Moreover, measurements from CODIF, HIA, and EFW should correlate. To ensure that the signatures in $\boldsymbol{E} \cdot \boldsymbol{J}$ are statistically significant, we also require that all ECRs are at least $100 \mathrm{~s}$ long, i.e., they are composed of more than 25 data points sampled every $4 \mathrm{~s}$. More details on the instrumentation and method can be found in H09A as well as a discussion of the appropriate frame of reference for the calculations of the power density.

\section{Observations and discussion}

In this article we use the same data base as in the companion paper H09A. The data base contains 116 identified CLRs and 35 CGRs in the plasma sheet as observed by Cluster in the summer and fall of 2001. Note that the automatic selection routine is constructed to select only the clearest and most typical ECRs from the available power density data. Hence, there might well exist more ECRs in the plasma sheet but which have not been selected by the automatic routine. In this article and in H09A, we only aim at identifying and studying the most distinct ECRs and therefore neglect less clear ECRs. For example, the occurrence frequency is hence most likely an underestimate. A more thorough discussion of the data base is presented in H09A.

\subsection{Life time}

Figure 2 shows the time extent, $\Delta T$, of the ECRs as observed by the Cluster spacecraft. Red corresponds to CLRs and blue to CGRs. The error bars indicate the uncertainty due to the limited statistics. Measurement errors are not included in the error bars. The plots are normalized so that the sum of all red bars is equal to one, and similarly for the blue bars. Note, however, that our automatic selection routine requires that $\Delta T \geq 100 \mathrm{~s}$ for an ECR to be accepted, hence no events shorter than $100 \mathrm{~s}$ are included in Fig. 2. This $100 \mathrm{~s}$ requirement is used for eliminating those events which may for example be too much contaminated with noise. We do not exclude the possibility of the existence of shorter events. However, to be on the safe side, due to noise in the data we do not include them in the statistics. See Appendix A in the companion paper H09A for a further discussion on the $100 \mathrm{~s}$ requirement (as well as additional demands which need to be fulfilled for an event to be classified as a CLR or a CGR).

As shown in Fig. 2, CLRs and CGRs that are shorter in time $(\Delta T)$ appear to be more common. Most ECRs are found to have $\Delta T$ of the order of about 1-10 min. Moreover, CGRs appear to be shorter than CLRs. Even though we cannot observe ECRs shorter than $100 \mathrm{~s}$, the tendency of the histogram bars being higher for shorter $\Delta T$, Fig. 2 suggests that the typical $\Delta T$ might well be somewhat smaller than $100 \mathrm{~s}$, in particular for CGRs.

The large scale MHD simulation presented in Birn and Hesse (2005) show that the generators in the boundary regions of the plasma sheet are not stationary in time. In Fig. 9 they present various characteristic quantities such as the cross-tail current density, the dawn-dusk electric field and the power density as a function of time. The power density shows oscillations between load and generator character with a period of about $4 \mathrm{~min}$. This makes approximately $2 \mathrm{~min}$ for loads and generators, respectively, which is approximately consistent with our result from Fig. 2.

However, a careful examination of the details of Fig. 9 in Birn and Hesse (2005) indicates in fact that the generator 


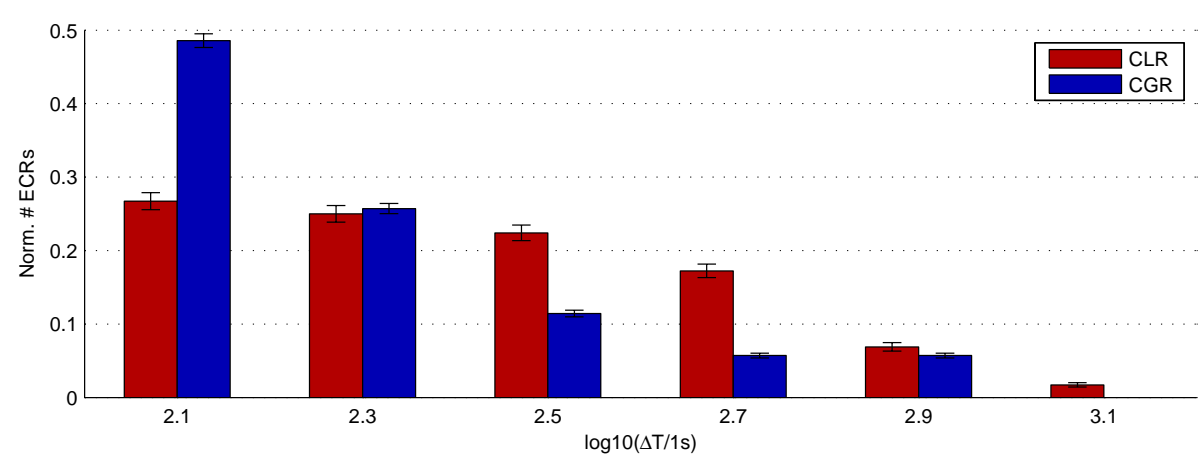

Fig. 2. Time extent of ECRs. Red and blue correspond to CLRs and CGRs, respectively. The error bars indicate the influence from the limited statistics (no instrumental errors are included). The data are normalized so that all red bars sum up to one and the same for the blue bars. We see that shorter CLRs and CGRs are more common. The cut-off at $\Delta T=100 \mathrm{~s}$ is caused by the selection criteria of the automatic routine.

regions extend over times $\sim 140$ s which are somewhat longer than the load regions $(\sim 100 \mathrm{~s})$. This is not supported by our detailed results. From Fig. 2 we instead note that CGRs, on the average, seem to be shorter than CLRs. Moreover, as discussed in the companion paper H09A, we observe more CLRs than CGRs in the Cluster data of the plasma sheet from 2001. An unequal amount of CLRs and CGRs does not agree with simple oscillations back and forth between $\boldsymbol{E} \cdot \boldsymbol{J}>0$ and $\boldsymbol{E} \cdot \boldsymbol{J}<0$. Furthermore, our results in H09A show that CLRs are stronger than CGRs, while panel three of Fig. 9 in Birn and Hesse (2005) on the contrary indicates that generator regions are stronger. It should be noted that Birn and Hesse (2005) is based on a MHD simulation which of course has inherent limitations. In addition to this, their results apply specifically to energy conversion regions close to the PSBL, whereas we investigate energy conversion in a cross section of the plasma sheet observed in the summer and fall of 2001, including both the PSBL and regions close to the neutral sheet. Consequently, the plasma sheet as observed by Cluster shows a more complicated picture than the one from Birn and Hesse (2005).

It is important to notice that more thorough investigations are needed to conclude if the $\Delta T$ obtained by Cluster directly corresponds to the actual life time of the ECRs. Such investigations will be presented in this article. If the ECRs are drifting, crossing the spacecraft path, $\Delta T$ will of course both be influenced by the life time of the ECRs and the time it takes for them to cross the spacecraft. To obtain a probable estimate of the ECR life time and scale size, we need to be able to separate between space and time variations in our data. Are the ECRs spatial entities, drifting over the satellites with negligible time variation? Or rather temporal entities, whose motion is negligible? Or something in between? We note at this point that the ECRs described in this article are essentially related to the plasma motion, as given by the estimate of the electric field as $\boldsymbol{E}=-\boldsymbol{V} \times \boldsymbol{B}$. We cannot observe ECRs in the plasma frame, since our electric field proxy vanishes in this reference system.
The Cluster mission offers for the first time the possibility of distinguishing between time and space variations. In this article we investigate the time and space variations of the ECRs and try to estimate typical values of their life time and scale size.

If the ECRs are spatial entities drifting over Cluster, a certain time shift should be visible in $\boldsymbol{E} \cdot \boldsymbol{J}$ between the satellites. Out of all 151 selected ECRs from the plasma sheet of 2001, only one single CLR appears to be clearly propagating over Cluster. We hence conclude that the ECRs in general are not propagating. The power density of this CLR is presented in the bottom panel of Fig. 3. To obtain the power density for each satellite, instead of using the average electric field, we have used the electric field from each separate spacecraft as discussed in Sect. 2.

As shown in the bottom panel of Fig. 3, apparently Cluster satellite $\mathrm{C} 3$ first encounters the CLR, followed by $\mathrm{C} 1$ and then $\mathrm{C} 4$. The order is the same when exiting the CLR: first $\mathrm{C} 3$, then $\mathrm{C} 1$ and finally $\mathrm{C} 4$. The time shift between the spacecraft is of the order of $10 \mathrm{~s}$. With a characteristic size of the Cluster tetrahedron of about $1500 \mathrm{~km}$, this corresponds to a velocity of the CLR of about $150 \mathrm{~km} / \mathrm{s}$.

We could not find another example of clear time shifts in the data base. Since the resolution of our power density data is $4 \mathrm{~s}$, we cannot accurately resolve time shifts shorter than about $4 \mathrm{~s}$, which corresponds to a drift velocity of about $400 \mathrm{~km} / \mathrm{s}$ (the characteristic size of the Cluster tetrahedron is approximately $1500 \mathrm{~km}$ ). Time shifts due to drifts larger than this are not possible to resolve. If the ECRs were propagating with a higher velocity, say for example the Alfvén velocity (on the average the Alfvén velocity is ten times larger than the plasma velocity for our events), this time shift would also not be possible to resolve. Note also that some of the more extended ECRs in the data base might well be composed of many overlapping smaller ECRs. Determining possible time shifts for such complex structures is impossible.

From a manual inspection of all 151 included events, we note that for a majority of those (more than $60 \%$ of the 

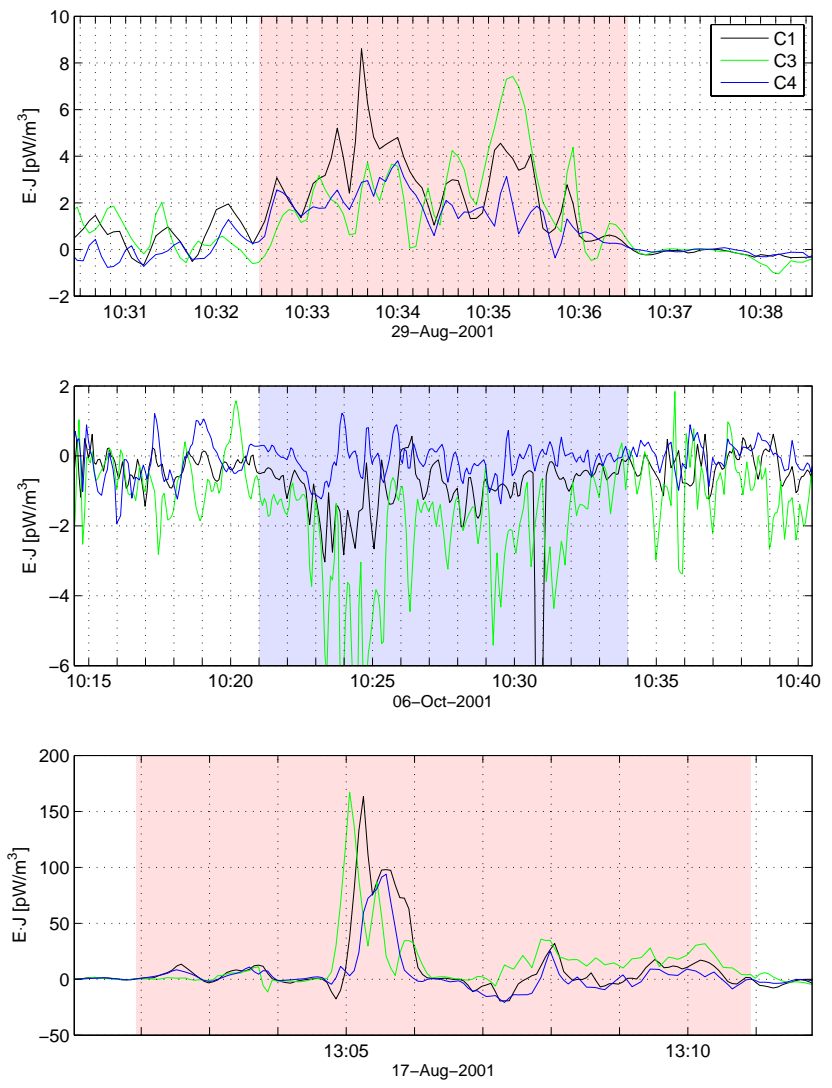

Fig. 3. Three examples of ECRs observed by Cluster from the 2001 plasma sheet data. (For examples of the electric field and current density signatures within ECRs, we refer to Fig. 2 in H09A.) Top panel: Typical example of a CLR where all three spacecraft are embedded inside the CLR and see approximately the same power density signatures. Middle panel: Typical example of a CGR. In this case $\mathrm{C} 4$ is outside the CGR and $\mathrm{C} 1$ is rather close to the edge. Bottom panel: The only event with a clear time shift observed in the data base. The CLRs (CGRs) are highlighted in red (blue). Note the different scalings of the power density and time axes. Especially the CLR in the bottom panel show large power densities around 13:05, making the nearby fluctuations seem insignificant even though they are well above the $0.4 \mathrm{pW} / \mathrm{m}^{3}$ threshold of the automatic selection routine.

events) there are no clear time shifts at all. Even though we do not visually observe any clear indications of time shifts for the remaining ECRs, we cannot use this manual inspection to unambiguously exclude time shifts, e.g. due to high velocity drifts for these events. Either the velocity was too high for us to be able to resolve any possible shifts, or the ECR structures were too complicated to be able to unambiguously match signatures on the different spacecraft and reveal any shifts.

To investigate the time shifts further, we have numerically compared the power density signals from pairs of satellites. To obtain the power density for each satellite, we have used the electric field from each separate spacecraft as discussed in Sect. 2. To estimate the time shift between the signal $f_{X}(t)$ obtained by satellite $\mathrm{X}$, and the signal $f_{Y}(t)$ obtained by satellite $\mathrm{Y}$, we compute the cross-correlation coefficient

$C_{X Y}(\tau)=\int \frac{d t\left[f_{X}^{+}-\left\langle f_{X}\right\rangle\right]\left[f_{Y}^{-}-\left\langle f_{Y}\right\rangle\right]}{\int d t \sqrt{\left\langle f_{X}^{2}\right\rangle-\left\langle f_{X}\right\rangle^{2}} \sqrt{\left\langle f_{Y}^{2}\right\rangle-\left\langle f_{Y}\right\rangle^{2}}}$,

where $f_{X}^{+}=f_{X}\left(t+\frac{\tau}{2}\right)$ and $f_{Y}^{-}=f_{X}\left(t-\frac{\tau}{2}\right)$.

The resulting time shift is obtained from the maximum value of the cross-correlation coefficient. Note that we cannot in general compute the time shift by using standard timing analysis and minimum variance methods due to the complicated geometry of the ECRs, and the highly varying power density signatures.

The result from the cross-correlation analysis is presented in Fig. 4. In preparing this figure, we have manually inspected all curves of $C_{X Y}(\tau)$ and removed those cases where one or two spacecraft are outside the ECR, hence making the cross-correlation unreliable. Moreover, for some ECRs, there are ambiguities in determining the maximum of the cross-correlation curve due to the existence of several significant local maxima. In those cases we have selected the one of the local maxima which is closest to a zero time shift.

In Fig. 4 we see that the most typical time shift is of the order of $0 \mathrm{~s}$, but there are also indications of possible time shifts $\gtrsim 4$ s. Note that there are cases when we, e.g., observe zero time shifts from two spacecraft pairs, and a $4 \mathrm{~s}$ time shift from another pair. This can be due to a complicated ECR structure or it can be caused by numerical errors in combination with noisy data. In these cases it is reasonable to assume that the true time shift in fact is smaller than $4 \mathrm{~s}$. Large time shifts in Fig. 4 are, at least sometimes, likely to be artificial and they are caused by highly variable and noisy signals. Therefore there is often no clear maximum of the $C_{X Y}(\tau)$ in these cases. The large time shifts are indeed mostly visible in the C13 and C34 panels, with C3 data known to be more noisy because of poorer count statistics, caused by degraded particle detection efficiency.

We conclude that a majority of the CLRs and CGRs observed in the plasma sheet at an altitude of about $15-20 R_{E}$ are not associated with a time shift of the order of $\gtrsim 4 \mathrm{~s}$ caused by a drift. There are two possible reasons for this. Either the ECRs drift much faster than the plasma bulk velocity (then we cannot resolve the drift), or the ECRs are rather stationary in space but varying in time, hence causing the appearance and disappearance of ECRs in the Cluster power density data. A detailed investigation of the ECR events is needed to analyze this. Below we therefore use our data base to further investigate time shifts.

Computing the power density by using the curlometer current for the entire Cluster tetrahedron but the electric field from each separate spacecraft (see Sect. 2), we can investigate how often the satellites are close to the edge of an ECR. Note that only $\mathrm{C} 1, \mathrm{C} 3$, and $\mathrm{C} 4$ are available when using the 

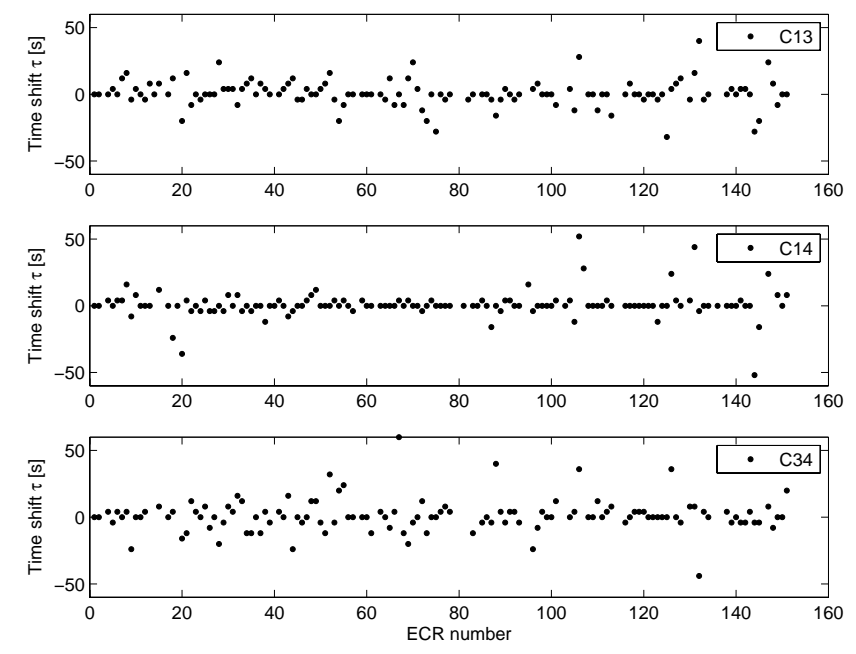

Fig. 4. The cross-correlation of power density signals from $\mathrm{C} 1$ and C3 (top panel), C1 and C4 (middle panel), C3 and C4 (bottom panel) is computed for each ECR according to Eq. (1). Numerically estimated time shift obtained from the maximum of the cross-correlation is plotted versus the ECR sequence number. We see that the most typical time shift is of the order of $0 \mathrm{~s}$, but $4 \mathrm{~s}$ and above is also possible.

electric field from CODIF. The top and middle panel of Fig. 3 show two rather typical ECRs from our data base. The ECR region is highlighted in red for the CLR and blue for the CGR. Later on in this article (see Sect. 3.2), we will show that details in the power density observed on the three spacecraft can be used to estimate the scale size of the ECRs. The black, green and blue curves correspond to estimates from $\mathrm{C} 1, \mathrm{C} 3$, and $\mathrm{C} 4$, respectively.

For the CLR in the top panel of Fig. 3, we see that all spacecraft appear to be inside the CLR. No apparent time shift is visible. Instead it seems that the CLR signature first grows and then decreases on all three spacecraft simultaneously. The CGR in the second panel of Fig. 3 extends over longer times $(\Delta T)$ than the one discussed in the previous paragraph. In contrast to the ECR in the top panel, this ECR shows evidence of the Cluster spacecraft being located closer to the edge of the ECR. In fact we believe that $\mathrm{C} 4$ is outside the ECR since it does not see any significant power density exceeding the surrounding fluctuations. Assuming that the ECRs investigated in this article correspond to regions with maximum power density in the center and smoothly decreasing power densities towards the boundaries, the signatures from the various satellites may offer an indication on their closeness to the boundaries. Comparing the magnitude of the power density variations observed by $\mathrm{C} 1$ and $\mathrm{C} 3$ in the second panel of Fig. 3, it is hence likely that C3 is deeper into the CGR while $\mathrm{C} 1$ is closer to the edge. This conclusion is based on the smaller magnitude of the power density observed by $\mathrm{C} 1$ as compared with $\mathrm{C} 3$. Note however, that more complex power density variations within the ECR than a single peak in the center may complicate the analysis.

For the event in the middle panel, the magnitude of the power density seems to grow and decrease simultaneously on all spacecraft similar to the CLR in the top panel. Moreover, if the ECR is drifting in space, we believe that it is rather unlikely that a single spacecraft (in this case $\mathrm{C} 4$ ) could be located outside the entire ECR while the other two are embedded inside it. Either the ECR is very small and does not reach $\mathrm{C} 4$ during the drift (but it cannot be too small since it is observed by two other spacecraft), or the ECR is rather stationary in space but varying in time causing the similar variations in the power density observed by the satellites $\mathrm{C} 1$ and $\mathrm{C} 3$. We consider the latter option being more likely, i.e., the CGR is probably rather stationary in space but varying in time.

Manual inspection of the ECRs in our data base show that the events displayed in the two top panels of Fig. 3 are rather typical. In more than half of the cases, we can manually exclude possible time shifts due to drifts over the spacecraft. Detailed investigations of the power density signatures from various spacecraft indicate that such drifts are negligible. In those events when a spacecraft is outside the ECR, we especially note that the spacecraft remains outside during the entire event. As discussed in the paragraph above, it is hence unlikely that such an ECR has a significant drift. Note, however, that both the direction of a possible drift as compared to the spacecraft positions, as well as the shape of the ECR in relation to the Cluster tetrahedron, can influence the size of possible time shifts.

We therefore conclude that a majority of the ECRs observed in the plasma sheet data from 2001 are rather stationary in space but instead varying in time. This conclusion is consistent with the simulation results presented in Fig. 9 of Birn and Hesse (2005), where we indeed see a time variation in the power density with oscillations between load and generator characteristics in the plasma sheet boundary region.

The prevalence of time variations for our ECRs implies that the time extent $\Delta T$ presented in Fig. 2 provides a proxy for the life time of the ECRs. Hence, the conclusion is that the ECRs investigated in this article have a life time of the order of 1-10 min. Moreover, the life time of CGRs are possibly somewhat shorter than for CLRs.

\subsection{Scale size}

A large data base offers advantageous possibilities of estimating the scale size, $\Delta S$, of the ECRs. The scale size estimate is based on information on the respective satellites' closeness to the edge of the ECRs. Manually going through our data base, we have identified all those CLRs and CGRs where one or more satellites are outside the ECRs. Moreover, we have manually also sorted out those events where one or more satellites are clearly closer to the edge (as for $\mathrm{C} 1$ in the CGR presented in the middle panel of Fig. 3). 


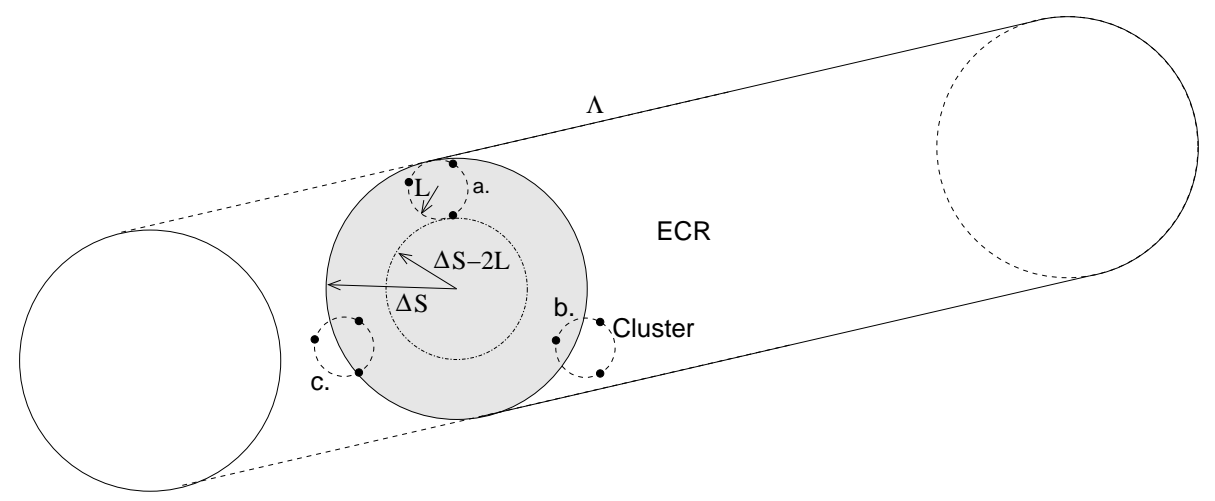

Fig. 5. Sketch of the overlap between a cylindrical ECR and the Cluster spacecraft. A cross-section of the ECR, containing three examples of overlapping spacecraft, is highlighted in grey. The radius of the ECR cylinder is $\Delta S$. $L$ is the characteristic size of the Cluster tetrahedron. A statistical investigation of overlaps between ECRs and Cluster are used for obtaining an estimate of the typical perpendicular scale size, $\Delta S$, of the ECRs cylindrical cross-section. The cylinder length along the axial direction, $\Lambda$, is probably much longer.

Out of our 151 ECRs, we find that 25 events have one or more spacecraft located definitely outside the ECR. Moreover, assuming that the ECRs correspond to regions with a peak in the power density close to the center and smoothly sloping power densities towards the boundaries, we find that there are in total 60 events with one or more satellites being close to the edge or outside the ECR. Only considering CLRs, the corresponding numbers are 14 and 38, respectively, and for CGRs 11 and 22. As discussed in H09A, in total there are 116 CLRs and 35 CGR in our data base.

For our plasma sheet data from the summer and fall of 2001, the Cluster tetrahedron is nearly equilateral with a planarity and elongation (Robert et al., 1998) around or below 0.1 . Therefore we can assume that the satellites are equally spread over the surface of a spherical volume in space. However, since CODIF is only operational on $\mathrm{C} 1, \mathrm{C} 3$, and $\mathrm{C} 4$, in reality we only use data from three spacecraft equally spread over the circumference of a circle. Assuming that the CLRs and CGRs are cylindrical, presumably aligned with the magnetic field, and of the same size, we can estimate the perpendicular scale size of the ECRs, $\Delta S$, from the observed overlap between the ECR cylinders and the Cluster satellites. The estimate of $\Delta S$ is hence obtained from analyzing the statistical variation of the cross sections of the ECRs and Cluster.

Figure 5 illustrates a few typical cases. In case (a) we see that all spacecraft are precisely inside the ECR cylinder whereas in (b) and (c) at least one spacecraft is outside. The radius of the ECR cylinder and the Cluster circle are $\Delta S$ and $L$, respectively. Strictly speaking, the projection of the inter-spacecraft separation should be used for the distance $L$. However, for practical purposes in the statistical analysis, we approximate this distance with the characteristic scale size of the Cluster tetrahedron. The probability $P$ of observing all spacecraft inside an ECR is dependent on the ratio between the volume of the inner cylinder with radius $\Delta S-2 L$ and the outer cylinder with radius $\Delta S$,
$P=\frac{(\Delta S-2 L)^{2}}{\Delta S^{2}}$.

Solving for $\Delta S$ we obtain

$\Delta S=\frac{2 L}{1-P^{1 / 2}}$,

and the typical ECR scale size can hence be obtained from $P$ which we estimate from our data base.

As discussed above, there are in total 151 ECRs which have one or more spacecraft inside the ECR, whereas 151-25=126 ECRs have all spacecraft inside the ECR. Using this we obtain a probability $P_{\mathrm{ECR}}=126 / 151$ and a typical scale size of the ECRs, $\Delta S_{\mathrm{ECR}} \approx 23 L$, where $L$ is the characteristic size of the Cluster tetrahedron. Performing the same calculation for CLRs and CGRs separately, we obtain $\left(P_{\mathrm{CLR}}=102 / 116\right.$ and $\left.P_{\mathrm{CGR}}=24 / 35\right)$ typical scale sizes of $\Delta S_{\mathrm{CLR}} \approx 32 L$ and $\Delta S_{\mathrm{CGR}} \approx 12 L$.

As discussed before, there are 25 events with satellites located clearly outside the ECRs, and 60 events if we also include satellites close to the edge. This information can be used to obtain upper and lower limits on the scale size $\Delta S$. Indirectly, the upper and lower limits may also indicate the dimensions of the ECR boundary regions on the assumption that the ECRs correspond to regions with a power density peak near the center, and decreasing power densities towards the edges. The estimate of $\Delta S$ in the previous paragraph is based on the assumption that there are only 25 events where Cluster probes the boundaries and regions exterior to the ECRs. Hence, this estimate corresponds to the upper limit on the ECR scale size. On the other hand, assuming that all 60 events are relevant for probing the boundary regions, we obtain a lower limit on $\Delta S$. In this case, we estimate the probabilities according to $P_{\mathrm{ECR}}=(151-60) / 151=91 / 151, P_{\mathrm{CLR}}=78 / 116$ and $P_{\mathrm{CGR}}=13 / 35$ for ECR in general and for CLRs and CGRs, respectively. The corresponding characteristic scale sizes are $\Delta S_{\mathrm{ECR}} \approx 9 L, \Delta S_{\mathrm{CLR}} \approx 12 L$, and $\Delta S_{\mathrm{CGR}} \approx 5 L$. 
It is probable that the ECR scale size is somewhere in between the above estimates of the upper and lower limits. Hence, in general the typical scale size in general is $9 L \lesssim \Delta S_{\mathrm{CLR}} \lesssim 23 L$, where $L$ is the characteristic size of the Cluster tetrahedron. In our data base of plasma sheet events from $2001, L \sim 1500 \mathrm{~km}$ and hence $2 R_{E} \lesssim \Delta S_{\mathrm{ECR}} \lesssim 5 R_{E}$.

For CLRs the corresponding scale sizes are $12 L \lesssim \Delta S_{\mathrm{CLR}} \lesssim 32 L$ or $3 R_{E} \lesssim \Delta S_{\mathrm{CLR}} \lesssim 8 R_{E}$. For CGRs we have $5 L \lesssim \Delta S_{\mathrm{CGR}} \lesssim 12 L$ or $1 R_{E} \lesssim \Delta S_{\mathrm{CGR}} \lesssim 3 R_{E}$.

Our estimate of the scale size, $\Delta S$, relies on the assumptions that the ECRs are equally big and cylindrically shaped with the cylinder axis along the magnetic field. In this article we have obtained the scale size perpendicular to the magnetic field. Note that we do not focus on the geometry of the ECRs, but only on the typical scale size. Other geometries may be possible and wave activity may complicate the interpretation of the data, but it is out of the scope of this article to work with more complicated structures. Moreover, obtaining an estimate of the ECR dimension along the magnetic field is an issue for future investigations. Furthermore, note that the scattering in the cross-correlation presented in Fig. 4 may well be due to the departure from a simple cylindrical geometry or due to noisy SC3 data - as already commented in Sect. 3.1. The general orientation of the Cluster tetrahedron, with $\mathrm{C} 3$ being located furthest to the south, may contribute as well to the different scattering in the $\mathrm{C} 13$ and $\mathrm{C} 34$ panels of Fig. 4, compared to C14.

The estimates of the ECR length scale and life time are based on the assumption that the satellites' closeness to the ECR edge can be probed by investigating the power density obtained from the curlometer current (valid over the entire Cluster tetrahedron) and the electric field measured by the individual spacecraft. However, as discussed in Sect. 4 of H09A, from a visual inspection we note that there is no statistical significant observation from our data base that variations in either the current density or the electric field should be more important than the other one for the resulting load or generator signature in the power density. Hence, our estimates of the length scale and life time apply, strictly specking, only for the ECRs which are dominated by the variation of the electric field, in the presence of a more slowly varying current density. We do not exclude, however, that they are valid for all ECRs.

Note that the cross-section between the satellite plane and the ECR cylinder generally is an ellipse, if the satellite plane is not normal to the cylinder axis. However, in this article we make the simplified assumption of a circular cross-section. Note also that some of our ECRs in the data base might well be composed of many smaller and overlapping ECRs. This naturally complicates the statistical analysis of the typical scale size. However, we believe that the obtained $\Delta S$ indicates the typical scale size of the ECRs.

The equal size of all CLRs and CGRs is of course a simplified assumption. However, as discussed in Marghitu et al. (2009), there are indications that ECRs are typically associ- ated with bursty bulk flows (BBFs) - although the reverse does not hold (BBFs not showing energy conversion are often seen). Therefore it makes sense to compare the ECR scale size obtained in this article with the typical dimensions of BBFs. Based both on statistical investigations and event studies, in Nakamura et al. (2005) it is shown that the typical size of the BBF flow channel in the $y$ direction is $2-3 R_{E}$, and somewhat smaller in the z-direction, $1.5-2 R_{E}$. This is consistent with our results. Therefore the assumption of equal size for ECRs may still produce reasonable conclusions.

At the altitude of about $15-20 R_{E}$ we can assume that the plasma sheet has dimensions of the order $30-40 R_{E}$ in the $\mathrm{y}$-direction and somewhat smaller in the z-direction. This is consistent with Fig. 5 in our companion paper H09A, where the size of the plasma sheet is mapped out in the GSM $y z$ plane by using the available data from the plasma sheet crossings in the summer and fall of 2001. A typical scale size of $2 R_{E} \lesssim \Delta S \lesssim 5 R_{E}$ of the cross-section of the ECR cylinders correspond to regions of the order of $10 \%$ of the plasma sheet in the yz-plane, i.e., a significant part of the plasma sheet.

As discussed in this article, we believe that the ECRs are rather stationary in space and with a life time $\Delta T$ of about 1-10 min. Similar to the simulation results presented by Birn and Hesse (2005), on time scales of the order of 1-10 min we believe that it is likely that the ECRs alternately rise and vanish in significant regions of the plasma sheet, perhaps mediated by Alfvén waves. One might speculate that a significant part of the ECRs observed at about $15-20 R_{E}$ in the plasma sheet are somewhat wavy in nature, locally oscillating energy back and forth instead of channeling it to the ionosphere. However, there are investigations which indicate that the picture is not that simple. As discussed by Marghitu et al. (2006) and Hamrin et al. (2006), some CGRs in the mid altitude plasma sheet may well be connected to the auroral ionosphere.

\section{Conclusions}

By examining the power density, $\boldsymbol{E} \cdot \boldsymbol{J}$, in this article we have investigated the scale size and life time of CLRs and CGRs observed by Cluster in the plasma sheet at an altitude of about $15-20 R_{E}$. In our investigations we have used the same data base as in the companion paper H09A. As discussed in H09A, more than 80 plasma sheet crossing from the summer and fall of 2001 are included in our data base of ECRs. An automatic event selection routine which implements a set of instrumental and physical requirements is used for identifying the CLRs $(\boldsymbol{E} \cdot \boldsymbol{J}>0)$ and CGRs $(\boldsymbol{E} \cdot \boldsymbol{J}<0)$.

We find that a majority of the ECRs observed in the high altitude plasma sheet are rather stationary in space. Within our data base, no clear time shift caused by a drift over the spacecraft below $\sim 400 \mathrm{~km} / \mathrm{s}$ is observed, except for one case (see the bottom panel of Fig. 3). We can therefore conclude 
that the observed time extent, $\Delta T$, presented in Fig. 2 is mainly influenced by the life time of the ECRs. We find that the life time is of the order of $1-10 \mathrm{~min}$, which is consistent with the result from the large scale MHD simulation of the plasma sheet and magnetotail presented in Birn and Hesse (2005). The life time of CGRs is somewhat shorter (perhaps even shorter than $100 \mathrm{~s}$ ) than for CLRs.

Assuming that the ECRs are of equal size and cylindrically shaped with the cylinder axis along the magnetic field, we have estimated the typical ECR scale size, $\Delta S$, perpendicular to the magnetic field. We cannot say anything about the ECR length along the magnetic field. In general, for the ECRs we find that $2 R_{E} \lesssim \Delta S_{\mathrm{ECR}} \lesssim 5 R_{E}$. Comparing CLRs and CGRs, we note that the CLRs are somewhat larger than CGRs. When comparing the scale size of the ECRs with the typical dimensions of the plasma sheet at an altitude of $15-20 R_{E}$, we note that the ECRs occupy a significant part of the plasma sheet. Summarizing, we believe that the ECRs observed here are rather stationary in space, but varying in time. On time scales of 1-10 min, ECRs rise and vanish in significant regions of the plasma sheet, possible oscillating between load and generator character. It is probable that at least some of the ECRs represent oscillations between load and generator character confined to the tail rather than involving any energy transfer to the ionosphere.

Acknowledgements. We thank the CIS team for the ion data, the EFW team for the electric field data, and the FGM team for the magnetic field data. O.M. acknowledges support through the PECS contract ECSTRA, C98048, and the kind hospitality of Max-PlanckInstitut für extraterrestrische Physik, Garching. We also thank K. Rönnmark for fruitful discussions and S. Buchert for help with the FGM data.

Topical Editor R. Nakamura thanks G. Rostoker and J. Vogt for their help in evaluating this paper.

\section{References}

Birn, J. and Hesse, M.: Energy release and conversion by reconnection in the magnetotail, Ann. Geophys., 23, 3365-3373, 2005, http://www.ann-geophys.net/23/3365/2005/.

Dunlop, M. W., Balogh, A., Glassmeier, K. H., and Robert, P.: Four-point Cluster application of magnetic field analysis tools: The Curlometer, J. Geophys. Res., 107, 1384, doi:10.1029/2001JA005088, 2002.

Escoubet, C. P., Fehringer, M., and Goldstein, M.: Introduction The Cluster mission, Ann. Geophys., 19, 1197-1200, 2001, http://www.ann-geophys.net/19/1197/2001/.

Hamrin, M., Marghitu, O., Rönnmark, K., Klecker, B., André, M., Buchert, S., Kistler, L. M., McFadden, J., Rème, H., and Vaivads, A.: Observations of concentrated generator regions in the nightside magnetosphere by Cluster/FAST conjunctions, Ann. Geophys., 24, 637-649, 2006, http://www.ann-geophys.net/24/637/2006/.

Marghitu, O., Hamrin, M., Klecker, B., Rönnmark, K., Buchert, S., Kistler, L. M., André, M., and Remè, H.: Cluster observations of energy conversion regions in the plasma sheet, in: Proceedings of the 15th Cluster workshop, Springer series on Astrophysics and Space Science Proceedings, in press, 2009.

Marghitu, O., Hamrin, M., Klecker, B., Vaivads, A., McFadden, J., Buchert, S., Kistler, L. M., Dandouras, I., André, M., and Rème, H.: Experimental investigation of auroral generator regions with conjugate Cluster and FAST data, Ann. Geophys., 24, 619-635, 2006, http://www.ann-geophys.net/24/619/2006/.

Nakamura, R., Baumjohann, W., Mouikis, C., Kistler, L. M., Runov, A., Volwerk, M., Asano, Y., Voros, Z., Zhang, T. L., Klecker, B., Balogh, A., and Rème, H.: Multi-point observation of the highspeed flows in the plasma sheet, Adv. Space Res, 36, 1444-1447, 2005.

Robert, P., Dunlop, M. W., Roux, A., and Chanteur, G.: Accuracy of current density determination, Chap. 16 in: Analysis methods for multi-spacecraft data, edited by: Paschmann, G. and Daly, P. W., ISSI Scientific Report SR-001, ISSI/ESA, Bern, 1998. 